\title{
Empyema caused by Optochin Sensitive Streptococcus mitis in the Course of Varicella
}

Sir,

Streptococcus mitis belongs to the viridans group of Streptococci and is traditionally regarded as a normal commensal of the oropharynx, skin, intestinal and genital tracts. ${ }^{1}$ We describe a case of empyema complicating varicella infection due to Streptococcus mitis in a child, a case not reported earlier to our knowledge.

A previously healthy 5-year-old boy with varicella infection presented seven days later to our emergency department with high grade fever, productive cough, dyspnoea and mild swelling of left side of chest. On admission, he had signs of respiratory distress, poor perfusion and severe dehydration. He was pale and dyspnoeic, with a temperature of $41.2^{\circ} \mathrm{C}$.The $\mathrm{PaO}_{2}$ was $66.5 \mathrm{~mm} \mathrm{Hg}$. Respiratory system examination revealed diffuse swelling, tenderness and increased temperature over left upper chest. The trachea was shifted to right side. Lung auscultation revealed dullness to percussion and decreased breath sounds over the left upper quadrant. Resolving lesions typical of varicella were seen on skin examination. The rest of the physical and neurological examination was normal. The white blood cell count was 13,400 cells $/ \mathrm{mm}^{3}(73 \%$ neutrophils, $25 \%$ lymphocytes, $1 \%$ monocytes, and $1 \%$ eosinophils), the hemoglobin was 10 $\mathrm{g} / \mathrm{dL}$, and the erythrocyte sedimentation rate was $35 \mathrm{~mm} /$ $1 \mathrm{st} \mathrm{hr}$. The chest radiograph showed complete opacity of left lung field, following which a diagnosis of left sided empyema was made and chest tube was inserted. About $1200 \mathrm{ml}$ of non-foul smelling blood streaked purulent fluid was aspirated. Patient was started on intravenous ceftriaxone $750 \mathrm{mg} 12$ hourly and amoxicillin-clavulanic acid 300mg 6 hourly alongwith oxygen and fluid management. Microscopic examination of the fluid showed 10-15 pus cells/oil immersion field and Grampositive cocci in chains, later on culture, a pure growth of a-hemolytic colonies was obtained on blood agar. The colonies were identified on the basis of Gram stain, catalase test, Optochin sensitivity \& bile solubility. ${ }^{1}$ Gram stain of the colonies revealed Gram-positive cocci. The Catalase test was negative. The colonies were optochin sensitive $(>22 \mathrm{~mm})$ in both ambient air and under $5 \% \mathrm{CO}_{2}$. and were bile insoluble. The Quellung test was negative. ${ }^{1}$ These colonies were characterized as viridans Streptococci and later biochemically identified as member of Streptococcus mitis group (SMG), (these a-hemolytic strains form acid in the presence of lactose but not in the presence of mannitol; did not produce sorbitol, acetylmethylcarbinol, arginine, and urease; and did not hydrolyze esculin). ${ }^{1}$ The strain was susceptible to penicillin, clindamycin, ampicillin + sulbactum, vancomycin, teicoplanin, linezolid and were resistant to gentamicin and amikacin. Linezolid $150 \mathrm{mg} 12$ hourly was added to the treatment after availability of culture report. Antibiotic treatment was continued for four weeks, leading to complete clinical and radiological resolution.

There are very few reports in the literature describing Opt sensitive (Opts) SMG. ${ }^{2-5}$ Hence, speciation of viridans streptococci when isolated in pure culture is a must for precise diagnosis. This case report is important that it shows a case of post-varicella empyema due to Streptococcus mitis and provides contribution to the literature regarding optochin sensitive Streptococcus mitis.

Anupam Das, Bijayini Behera ${ }^{1}$, Molly Madan, Purva Mathur ${ }^{1}$ and Anita Pandey Department of Microbiology, Subharti Medical College, Meerut, Uttar Pradesh, India

${ }^{1}$ Department of Laboratory Medicine, Jai Prakash Narayan Apex Trauma Centre, All India Institute of Medical Sciences, New Delhi-110029 and

E-mail:purvamathur@yahoo.co.in [DOI-10.1007/s12098-010-0024-2]

\section{REFERENCES}

1. Winn W, Allen S, Janda W et al (eds). Gram positive Cocci, Part II. Streptococci, Enterococci, and the Streptococcus-Like Bacteria. Kaneman's Color Atlas and Textbook of Diagnostic Microbiology, 6th ed. Lippincott Wiliams and Wilkins, 2006; 725.

2. Borek AP, Dressel DC, Hussong J, Peterson LR. Evolving clinical problems with Streptococcus pneumoniae: increasing resistance to antimicrobial agents, and failure of traditional optochin identification in Chicago, Illinois, between 1993 and 1996. Diagn Microbiol Infect Dis 1997;29:209-214.

3. Martýnn-Galiano AJ, Balsalobre L, Fenoll A, de la Campa AG. Genetic characterization of optochin-susceptible viridans group streptococci. Antimicrob Agents Chemother 2003; 47: 3187-3194.

4. Mundy LS, Janoff EN, Schwebke KE,Shanholtzer J,Willard KE. Ambiguity in the identification of Streptococcus pneumoniae. Optochin, bile solubility, quellung, and the AccuProbe DNA probe tests. Am J Clin Pathol 1998; 109: 55-61.

5. Balsalobre L, Hernández-Madrid A, Llull D et al. Molecular characterization of disease-associated streptococci of the mitis group that are optochin susceptible. J Clin Microbiol 2006; 44: 4163-4171. 